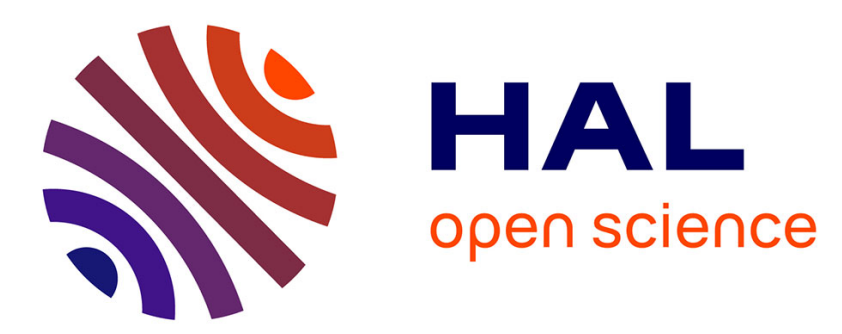

\title{
Designing Interaction in Virtual Worlds through a Passive Haptic Peripheral
}

Jean-Rémy Chardonnet, Jean-Claude Léon

\section{To cite this version:}

Jean-Rémy Chardonnet, Jean-Claude Léon. Designing Interaction in Virtual Worlds through a Passive Haptic Peripheral. 2012 IEEE RO-MAN: The 21st IEEE International Symposium on Robot and Human Interactive Communication, Sep 2012, Paris, France. pp.284-289, 10.1109/ROMAN.2012.6343767 . hal-00778150

\section{HAL Id: hal-00778150 https://hal.science/hal-00778150}

Submitted on 18 Jan 2013

HAL is a multi-disciplinary open access archive for the deposit and dissemination of scientific research documents, whether they are published or not. The documents may come from teaching and research institutions in France or abroad, or from public or private research centers.
L'archive ouverte pluridisciplinaire HAL, est destinée au dépôt et à la diffusion de documents scientifiques de niveau recherche, publiés ou non, émanant des établissements d'enseignement et de recherche français ou étrangers, des laboratoires publics ou privés. 


\title{
Designing Interaction in Virtual Worlds through a Passive Haptic Peripheral
}

\author{
Jean-Rémy Chardonnet ${ }^{1}$ and Jean-Claude Léon ${ }^{2}$
}

\begin{abstract}
This paper presents a prototype of a hands-on immersive peripheral device for controlling a virtual hand with high dexterity. Based on the results of users' tests on previous versions of our device and on the analysis of a manipulation task, this prototype is as easy as a mouse to use and allows the control of a high number of degrees of freedom (dofs) with tactile feedback. Design issues, physical phenomena and physiological behaviors are tightly linked and highly influence interaction. The goals corresponding to these issues include the choice of sensors' technology and their position on the device, low efforts exerted while using the device, relevant multisensorial feedback, performance of achieved tasks. An example of a grasping task illustrates the effectiveness of our device to achieve intuitive and efficient interactions, bringing new insights for collaborative interaction.
\end{abstract}

\section{INTRODUCTION}

Object manipulation is a natural and essential everydaylife task that can be done alone or in collaboration with other persons or robots. Manipulating an object is conditioned by the number and the quality of information returned to the brain. This paper presents an extension of the HandNavigator, an immersive peripheral for controlling a virtual hand in virtual environments [1] for precise object manipulation.

\section{A. Related work}

Interacting with an object is a difficult task to achieve since several possible grasping configurations are possible. Thus, imitating a grasping motion in virtual environments is not easy. This diversity of configurations, conditioned by several parameters such as the shape, is not addressed by the current devices and software. Indeed, each movement of a hand and its fingers cannot be controlled in virtual environments because the interfaces complexity dramatically increases. Nevertheless, some natural configurations of a hand can be achieved as long as interfaces give enough immersive sensations. Through an appropriate peripheral device allowing some motions of a hand, it is possible to evolve from a qualitative validation of a task to a quantitative one where hand configurations can be formalized and quantified.

Several solutions have been proposed to perform general manipulation tasks in virtual environments, transforming motions of a real hand to motions of a virtual one [2]. Often, corresponding solutions rely on motion capture based systems, for instance optical systems through cameras [3],

\footnotetext{
${ }^{1}$ J.-R. Chardonnet is with Arts et Métiers ParisTech, CNRS, Le2i, Institut Image, 71100 Chalon-sur-Saône, France jean-remy . chardonnet at ensam.eu

${ }^{2}$ J.-C. Léon is with Grenoble University, INRIA, Jean Kuntzmann Laboratory, 38041 Grenoble Cedex 9, France jean-claude.leon at grenoble-inp.fr
}

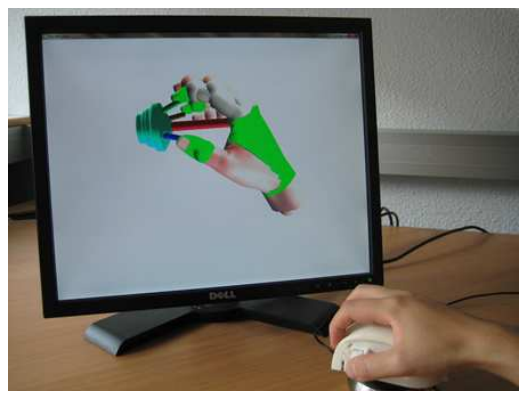

Fig. 1. Prototype of the HandNavigator integrating a tactile feedback.

[4], or mechanical systems through data gloves where sensors are attached to an exoskeleton covering the hand [5]. These solutions allow a high number of possible configurations of a hand, thus generating multiple possibilities to move a hand at real scale around the objects and, consequently, to grasp and manipulate them. However, they have several major drawbacks. These systems require preliminary calibration, which can be time consuming. Their capability to generate a large range of motions drastically increases their mechanical complexity and hence, their cost. As mentioned above: calibration is complex but using such systems is also difficult. Consequently, these systems are not ready-to-use. More precisely, it is difficult to load pre-saved configurations or to analyze them for issues related to accessibilities or trajectories, e.g., for collaborative tasks. Also, it is not possible to interrupt, at any time, a current task without losing its parameters and current configuration. Contact is hard to interpret even with some feedback, especially in the case of continuous contact, e.g., sliding a finger on a surface without loosing contact. Optical based systems suffer from occlusion problems. Indeed, there is often a point that is not visible by the camera. To solve this issue, cameras can be added but cost and space highly increase without avoiding configurations where occlusion problems remain. From an ergonomic point of view, achieving long tasks with a raised hand configuration quickly generates a muscular fatigue.

Simple systems, designed to be integrated in desktop environments, have been proposed, such as the SpaceNavigator, to achieve 3D interactions. They allow a maximum of six degrees of freedom (dofs) and a comfortable use because the user does not use raised hand configurations. These devices are well suited for pointing and object moving tasks. Their main limitation comes from their number of dofs that is not appropriate for more complex manipulation tasks requiring more dexterity, as required for example for col- 
laborative tasks with other avatars (humans, robots). Multitouch pads are more and more used in laptop computers, smartphones and allow more complex interaction with virtual environments [6], [7]. Their main advantage lies in their high sensitivity, allowing a good ergonomics for long tasks (from tens of minutes to one hour). However, they are designed mainly for 2D applications. Recently, some 3D applications are emerging but they cannot be enough immersive because these interactions are still performed in 2D.

Some systems integrate a haptic feedback [8], [9]. This feedback can also be found on larger equipment, such as haptic devices used for instance to interact in physical simulations (see for example [10], [11]), and includes a force feedback. These solutions increase the amount of information sent to the user and thus allow to better take advantage of the sensory capabilities of his/her brain. Haptic feedback is perceived only when the user touches a virtual object but is lacking totally or partially when moving freely in 3D space, without any contact [12], [13]. Active haptic systems' complexity increases as the number of force feedback components increases. These systems allow a user to better feel the virtual objects, but are hardly used by the general audience as they are expensive and they have a technological complexity that needs a good knowledge of the device. Moreover, one major limitation is parameters tuning, required to get realistic sensations of physical models, which is time-consuming and purely subjective.

One last solution is to provide passive feedback. This feedback can be perceived as a improvement compared to systems without any feedback [14], and even if it does not allow a user to get a total immersion in virtual environments, it can fool the user's proprioceptive senses [15] at low cost by using simple objects such as sponges or vibrators [13], [16]. It can easily be integrated in classical peripheral devices.

\section{B. General presentation}

Regarding the approaches above, our solution is based on a passive haptic feedback and a hands-on interaction. We present an extension of the HandNavigator described in [1]. Especially, validation tests of the existing prototypes are conducted by several users on grasping tasks scenarii, leading to the design of a new requirements of the HandNavigator with a more ergonomic shape, sensors allowing better dexterity and interactions, while integrating tactile feedback for an enhanced immersion, which is not the case in [1].

In this paper, we will at first expose the issues related to different possible configurations for object manipulation. Then, we introduce the previous prototypes and their results of validation tests performed on these prototypes, these results are analysed for the design of a new version. The following section describes the new prototype and shows a simple example using this prototype before concluding.

\section{ISSUES AND GOALS}

The kinematic structure of our hand allows us a wide range of configurations, among which we select the best one to perform the desired task. This ability to adapt the configurations to objects greatly improves our dexterity. Dexterity reduces here as the coordination between a hand, its fingers and the user's eyes, implying the ability to use them to perform activities requiring high accuracy. Thus, visual and tactile feedbacks are mandatory to achieve fine dexterity [17]. More precisely, the concept of dexterity here is bounded by a group of tasks we want to perform with our peripheral device. These tasks can be summarized as follows:

- contact-free motion of each virtual finger independently or not from the others, as natural as possible;

- manipulate objects as naturally as possible.

To achieve them, we must focus on the kinematics allowing a user to control independently the global motion of his virtual hand and that of its fingers. The simultaneous mobility of a hand and its fingers is mandatory to perform a large panel of tasks when a user interacts with the device. When analyzing a real grasping motion, we can distinguish three steps:

1) the hand and its fingers move freely in $3 D$ space to reach the object to be grabbed. During this step, the user already adapts the configuration of his hand to fit the object's shape;

2) the hand and its fingers touch the object. Contact can appear sequentially (the hand moves towards the object then its fingers bend to touch it) or progressively (the hand moves while closing its fingers). During this step, tactile and visual feedbacks are necessary and guide the user while performing this task;

3) the user applies a force on the object to grip it. This force depends on the friction between the object and the hand and its fingers. For instance, the low friction of slippy objects requires higher gripping forces so that the objects do not slip. Here also, kinesthetic and touch feedbacks guide the user to act on the object in the best way. During this step, his hand configuration evolves, thus generating different hand's postures.

To perform these steps, the user controls a high number of dofs. In virtual worlds, several dofs of a virtual hand mechanism can be configured using inverse kinematics, allowing a user to generate a large number of postures while reducing the number of dofs to control on the device. However, it is mandatory to monitor more than six dofs (defining the global hand position and orientation) to perform precise tasks with high dexterity and one dof per finger is a minimum.

Considering these characteristics, the device we develop must take into account the following requirements: (i) allow the user to control simultaneously a high number of dofs, (ii) allow a user to perform complex hand and finger motions, (iii) ergonomics to avoid pain or fatigue that drastically reduces motion dexterity and does not allow a long use (from several minutes to several hours), (iv) return to the user relevant contact information, and (v) be cheap, easy and ready to use, and calibration-free to ease its integration.

\section{ANALYSIS OF THE EXISTING PROTOTYPES}

\section{A. Existing prototypes and users' tests}

We describe the main features of different prototypes of the HandNavigator and users' tests performed on these proto- 

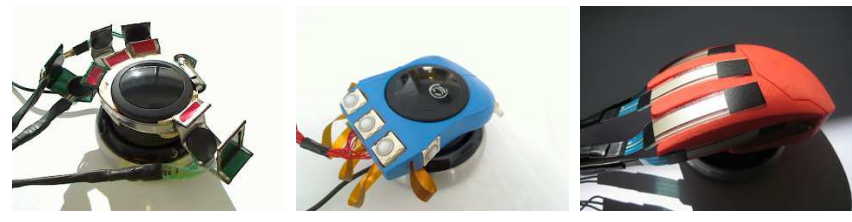

Fig. 2. Existing prototypes (from left to right: V2, V3t, and V3s).

types. The HandNavigator consists in two major components: a $3 \mathrm{D}$ mouse SpaceNavigator ${ }^{1}$ and, on top, a housing where sensors for fingers are located. The prototypes presented in [1] use either pressure sensors, trackballs, or scrollpads (see Fig. 2). We refer the reader to [1] for a detailed description of the SpaceNavigator and the different versions of the HandNavigator. We call these prototypes V2, V3t, and $\mathrm{V} 3 \mathrm{~s}$ respectively, according to their chronology.

To measure the performance, the usability, the controllability of each prototype and validate issues linked to human perception and learning capabilities, we performed tests with unexperimented users on each prototype. With each prototype, each user followed the procedure hereunder:

1) the user manipulates the devices for three minutes. Two sub-tasks are proposed:

- firstly, he concentrates on the motion of the virtual hand and tries to reach a green sphere fixed in the virtual scene. A yellow sphere, attached to the virtual hand, helps the user visualizing a target area on this object. When the two spheres meet each other, the green one changes color to indicate that the global hand position is satisfactory;

- then, the user concentrates on the motion of the virtual fingers. The virtual hand stands still in the virtual scene to allow the user acting freely on the sensors to monitor the virtual fingers, without generating any perturbation on the SpaceNavigator in charge of the global hand position;

2) subsequently, the user grasps a virtual giraffe located at some position in the virtual scene (see Fig. 3). The user gets feedback when the giraffe is grasped, since it changes color. The user must repeat this task several times. During each trial, the time required to achieve this task is measured to get a learning curve of the user's adaptation to the different prototypes.

At the end of the test, users had to fill up the questionnaire reproduced in Table I. The users score each criterion from 0 to 4 . Note that, compared to other questionnaires of the literature, e.g., [18] or the NASA-TLX protocol [19], devoted to task achievement evaluation, we adapted their content to evaluate our prototypes. We asked 32 users aged between 20 to 25 , right-handed, left-handed, men and women, to perform these tests. We obtained the results depicted in Fig. 4.

We observe that the prototypes get better scores as they evolve, especially in terms of comfort and usability, the users feel less pain or fatigue with the last prototype V3s, with

${ }^{1}$ http: / / www. 3dconnexion.com

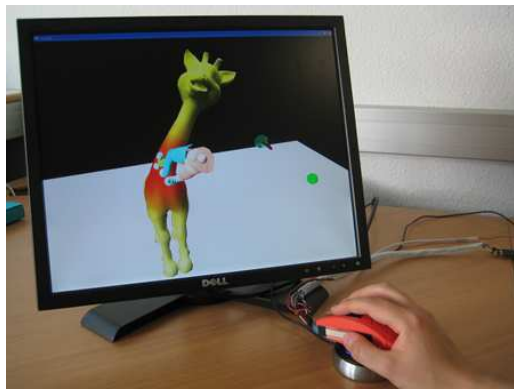

Fig. 3. Grasping a virtual giraffe.

TABLE I

QUESTIONNAIRE FOR EVALUATING EACH PROTOTYPE.

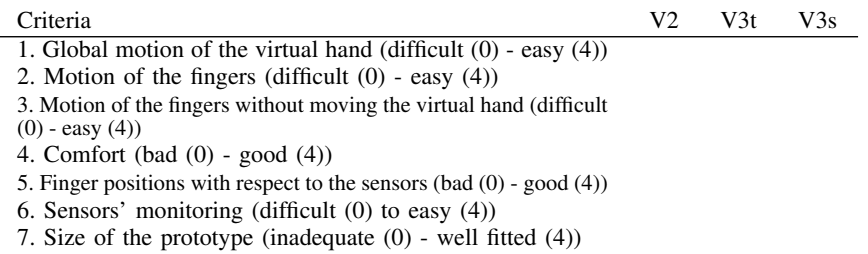

improved performance. However, we found out that the users had difficulties to move the virtual fingers without moving the virtual hand because they held the device too tightly. We think that it is a psychological phenomenon due to the novelty of the device that causes stress, exactly in the same way people using a computer mouse for the first time. An extended use shows that this stress tends to disappear.

The learning curve for each prototype is depicted in Fig. 5. We observe an adequacy between the qualitative results in Fig. 4 and the required completion time. The prototype V3s is easier to control because after only three trials the users can grasp the giraffe in less that $5 \mathrm{~s}$ whereas with the first prototype: V2, the time does not reach less than nearly 20s. Note that the prototype V3s seems to be the most intuitive and the one the users are immediately the most familiar with.

\section{B. Discussion and new design requirements}

The users' tests teach us several important issues allowing us to design a new prototype that is more efficient in terms

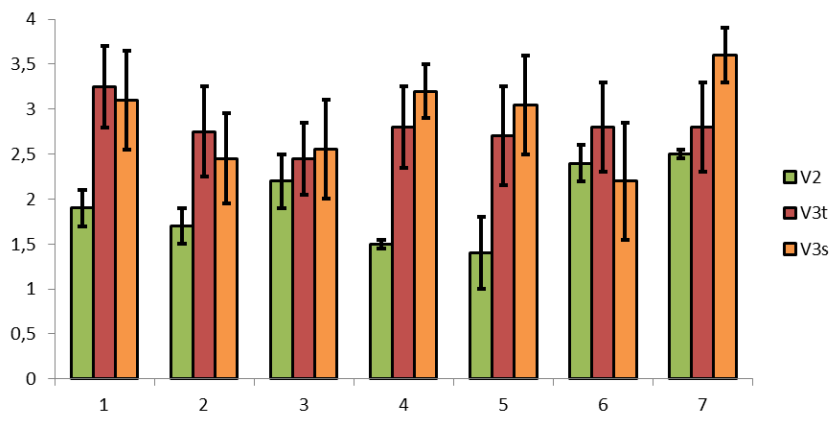

Fig. 4. Evaluation of the prototypes. The numbers along the $\mathrm{X}$ axis are the identifiers of the criteria listed in Table I. 


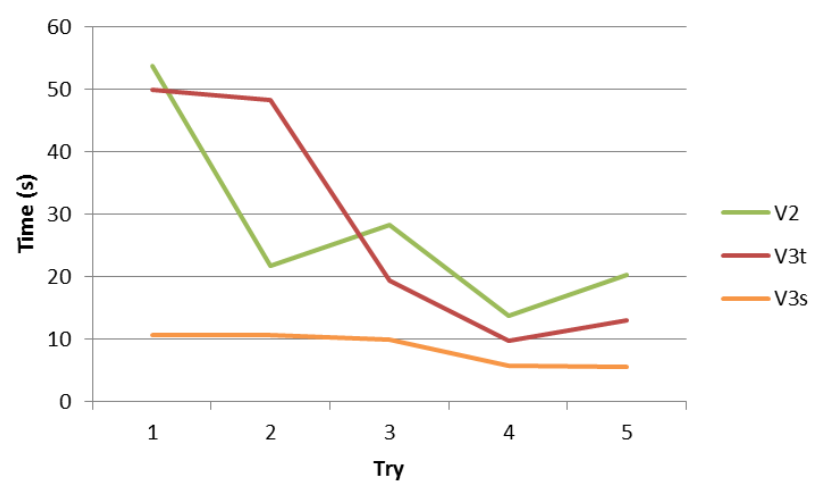

Fig. 5. Learning curves for each prototype in the scenario of Fig. 3. The curves do not evolve significantly after five trials.

of interactivity, dexterity and comfort.

The first issue is technological and addresses the sensors used to control the finger motion. To be efficient and avoid user's fatigue and undesired motions, we should consider a range of sensors allowing continuous motion of the real fingers between the open and closed configurations of the virtual fingers. One of the main reasons for which prototype V2 is badly evaluated by the users is the latency in finger motion: there are two sensors for each finger, one to open and one to close it. V3t uses trackballs and does not exhibit latency when opening/closing the real fingers because the real fingers just rotate slightly the sensor to switch from virtual finger's opening to closing. However, the user must roll them several times to reach the desired finger configuration, which, for long tasks, can be painful. V3s uses scrollpads and allows the user moving continuously and smoothly the virtual fingers. The major difficulty with these sensors is their constraint set on the user's fingertips having to travel a long distance to generate large virtual finger motions, increasing the probability of producing undesired motions. As a conclusion, it appears important to identify sensors capable of producing a high ratio of virtual finger to real finger displacement. Therefore, we will consider another sensor's technology: lever-switches, that can be found for example on digital cameras to zoom in or out. If the user releases the sensor, it goes back to its neutral position and the virtual finger stops.

The second issue relates to neuro-psycho-physiology. The interest of relying on small motions of real fingers to produce large motions of the virtual fingers is not only mechanical: it avoids solliciting too much the user's muscles of his arm and forearm. We observed during the evaluation tests that users were stressed on the prototypes. When reducing their muscular activity, we ensure them a better dexterity and performance, coupled with better comfort of use. Besides, generating full scale motions of the hand and the fingers is useless, as obtained with data gloves for example. On the one hand, with the sensors' technologies used, we can get a fine enough dexterity and accuracy. On the other hand, several neuro-psychological studies show that for a same task, between motor activity (the effective task) and mental activity (the pure cerebral activity of a task), very small difference is observed in terms of duration [20], [21]. In other words, if the user, without moving his hand and fingers, performs a pure cerebral task, he can get roughly the same performance results as if he is really performing the motion while producing a very small muscle activity. Finally, other studies show that for tasks requiring high precision and dexterity, the hand is the most efficient limb of the human body. Indeed, the fingers, more specifically the thumb, and the hand occupy the largest area of the somatosensorial cortex compared to other limbs [22], [23]. Thus, it is important to focus specifically on the way to use the device to increase performance and dexterity.

The last issue focuses on the tactile feedback when contact occurs between the virtual hand and an object. Indeed, just looking at an object is not enough, especially in virtual worlds, to determine the way to grasp it because parameters such as friction, temperature cannot be sensed if there is no contact, and occlusions can appear. In the evaluation tests, none of the prototypes include a tactile feedback, which can explain the time required to grasp the giraffe (at least 5s) whereas it is initially close $(20 \mathrm{~cm})$ to the virtual hand. In real conditions and at equal displacement speed, it should take only one second to grasp it. Therefore we will set up a passive haptic feedback, enough to fool the users's proprioceptive senses.

We see that the design of an immersive device goes beyond pure mechatronics and must also consider more general issues linked to neuro-psycho-physiological activities.

\section{NEW PROTOTYPE STRUCTURE}

\section{A. Individual module}

In order to incorporate the different requirements mentioned above, we designed a module allowing the user to control just one finger. This module is depicted in Fig. 6. It contains a lever-switch, for controlling a virtual finger with a low motion amplitude of a real finger, a pressure sensor and a vibrator to give the user touch and grasp feelings. The lever-switch is active only when the finger is free to move in 3D space, i.e., without any contact. The virtual finger is velocity-based controlled, allowing a user to interrupt and resume a task anytime without loosing its configuration. The vibrator is activated only when the virtual finger touches a virtual object and vibrates for a short time (half a second). The pressure sensor aims at giving the sensation to the user that he really grasps a virtual object and it is active only while the user is holding it. Indeed, the pressure sensor has a lower sensitivity than the other sensors we incorporated [1], as the user must apply higher forces $(1 \mathrm{~N})$ than to the SpaceNavigator $(0.4 \mathrm{~N})$ to activate it. Through this intrinsic characteristic, the user has to apply a significant force, similar to a configuration he encounters when holding tight a real object. Finally, a spring between the lever-switch and the pressure sensor separates the two modes (free and constrained hand motions). 

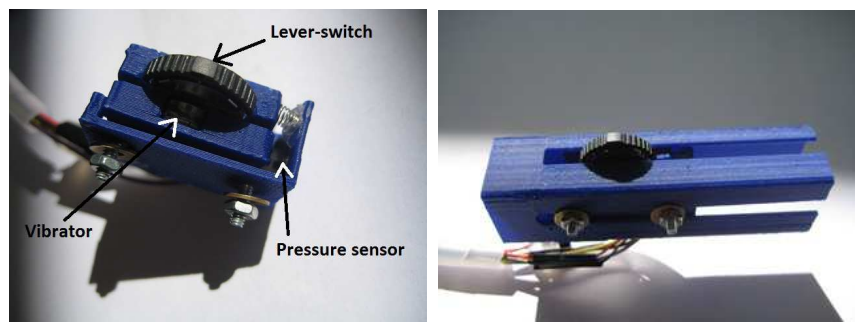

Fig. 6. Module for one finger containing the lever-switch and pressure sensors and a vibrator.
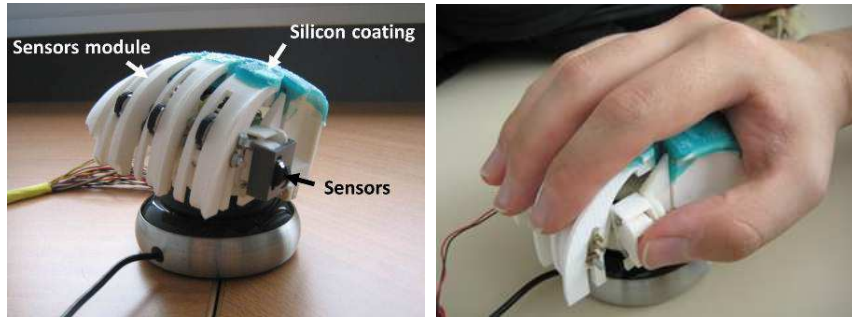

Fig. 7. Final prototype V4.

Finally, since one of our requirement devotes the device to a general audience, each module is inserted in an armhole so that the module can be adapted to a large range of hand sizes. The interest of separating the module from the housing is not only ergonomics, it enables setting up vibration barriers to avoid their propagation in the whole structure, which will be of major concern for the final prototype.

\section{B. Complete prototype}

We duplicate the module to include the other fingers. We completely redesigned the shape of the prototype to be ergonomic and allow better interaction. Similarly to the previous prototypes and for the same reasons related to the hand kinematics, it is possible to control only four fingers. Each finger has one sliding module for different hand sizes. When a user touches an object, he can immediately know which finger touches the object, thanks to individual vibrators. The final prototype, called V4, is depicted in Fig. 7. The schematic design of the prototype is shown in Fig. 8. As for the previous prototypes, the SpaceNavigator allows the control of the 6 dofs of the virtual hand's wrist. Each module controls the joint position of the last phalanx of a virtual finger. The joint position of the other phalanxes are equal to the one of the last phalanx to simplify the device. We used laser sintering techniques to manufacture this prototype and added a silicon-based coating as damping material to filter out the vibrations and isolate each finger.

\section{DEMONSTRATION}

We now show an evaluation of prototype V4 through a task example. The goal is to position the virtual hand and fingers on a part coming from a food processor as a manual assembly task. As shown in Fig. 9, there are several possible configurations to grasp it. The virtual hand's postures shown on bottom side of Fig. 9 were performed using prototype

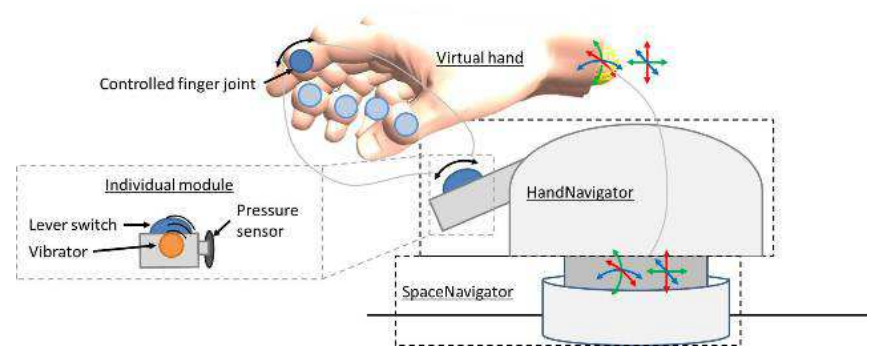

Fig. 8. Schematic design of the final prototype V4.
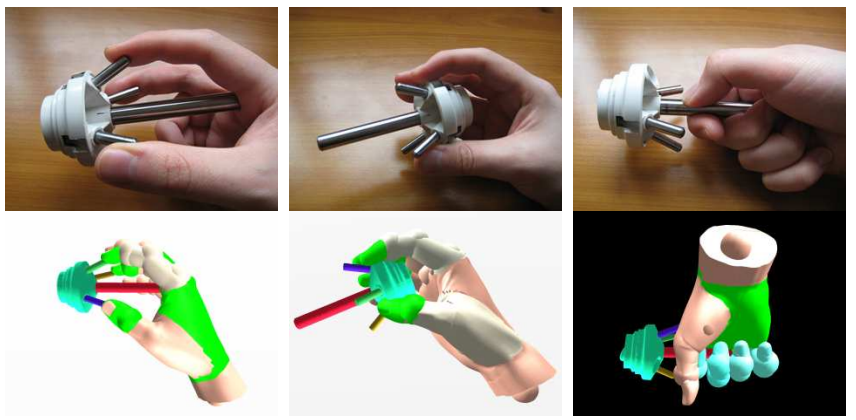

Fig. 9. Different possible configurations of virtual/real hands and fingers to grasp an object.

V4. We see that for the last configuration, we do not have exactly the same configuration as in reality, especially for the thumb. Indeed, we have only one dof for bending and extension as a planar movement. Thus, it is not possible to reflect the adduction/abduction movement of a real thumb. To solve this problem, we have to integrate bio-mechanical based kinematics models of the hand.

For the two other cases, we can see that the virtual configurations are very close to the real ones. We added some visual markers on the virtual hand to help the user, especially when the virtual hand or a finger touches an object. Even if the vibrations sensed by the user allow him to better reach the desired configurations, adding this visual feedback increases also the user's dexterity. We measured the time needed to reach the first configuration of Fig. 9 in configurations where there is no visual and tactile feedbacks, tactile feedback only, visual feedback only and, finally, both feedbacks. Considering the virtual hand moves at $0.2 \mathrm{~m} . \mathrm{s}^{-1}$ and the object is at an initial distance of $70 \mathrm{~cm}$ from the virtual hand, we get the following times respectively: $24 \mathrm{~s}$, $12 \mathrm{~s}, 12 \mathrm{~s}$ and $10 \mathrm{~s}$. Note that in reality, at equal displacement speed and distance, we get a time of $7 \mathrm{~s}$. We can clearly see the interest of having multi-sensorial feedback as, for the same task, we double the user performance between the cases without any feedback compared to both feedbacks. Indeed, without any visual or tactile feedback, it is necessary to modify the camera viewpoint to ensure a correct finger position. We observe that tactile feedback only does not bring more dexterity than just the visual one, probably because the user relies on visual markers, and we are in a configuration without any occlusion problem. However, the combination of both visual and tactile feedbacks brings advantages. Finally, 


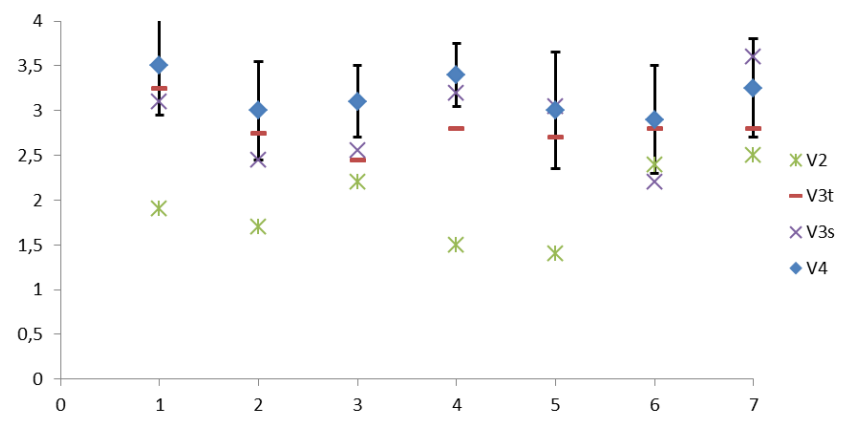

Fig. 10. Evaluation of the prototype V4. The numbers along the $\mathrm{X}$ axis correspond to the criteria listed in Table I.

if we compare with the prototype $\mathrm{V} 3 \mathrm{~s}$, which does not provide any tactile feedback, for the same task, we get $29 \mathrm{~s}$ and 19s respectively. The times observed are greater than those with the prototype V4 because, as mentioned earlier, the sensors on the prototype $\mathrm{V} 3 \mathrm{~s}$ require higher forces that can generate undesired motions. Based on this comparison, we show the interest of the sensors used in the prototype V4.

Our new device significantly improves the interactions, thanks to the simultaneous use of several dofs, allowing more complex tasks with virtual avatars for example.

Finally, we also carried out the same users' tests with our new prototype. We obtained the results depicted in Fig. 10, which confirms the improvement of our prototype.

\section{CONCLUSIONS}

We described and analyzed a prototype of an immersive peripheral device allowing a user to control a virtual hand and its gestures. The analysis of users' tests on previous versions of the device originated the design of this new version improving the control of the virtual hand postures as well as the dexterity to manipulate virtual objects, thanks to multi-sensorial feedbacks (tactile and visual). We especially concentrated on including tactile feedback to improve the user's immersion and interaction quality. Studies on the device shape, sensors' technologies, led to issues where physical phenomena, psycho-physiological behaviors as well as the device structure influence significantly the global interaction between real and virtual worlds. Thanks to the combination of several sensors' technologies, our prototype is able to provide relevant tactile feedback, while being comfortable. As for the previous versions of the prototype, our new version is cheap, easy to integrate in desktop environments and is calibration-free.

The next step is to set up more experiments where muscular activities of the user's arm and forearm are measured so that our prototype can be validated in a quantitative way.

The HandNavigator was designed to be integrated in several applications, such as physical simulation, industrial processes, teleoperation of robots or to communicate with virtual avatars. We will consider these applications in a near future to bring new interaction capabilities.

\section{REFERENCES}

[1] J.-R. Chardonnet and J.-C. Léon, "Design of an immersive peripheral for object grasping," in ASME International Design Engineering Technical Conferences \& Computers and Information in Engineering Conference (IDETC/CIE), Montréal, Canada, August 15-18 2010.

[2] D. A. Bowman, E. Kruijff, J. J. Laviola, and I. Poupyrev, $3 D$ User Interfaces: Theory and Pratice. Addison-Wesley Educational Publishers Inc., 2004.

[3] G. Dewaele, F. Devernay, and R. P. Horaud, "Hand motion from 3d point trajectories and a smooth surface model," in European Conference on Computer Vision, 2004, pp. 495-507.

[4] M. Schlattman and R. Klein, "Simultaneous 4 gestures 6 dof realtime two-hand tracking without any markers," in ACM symposium on Virtual Reality Software and Technology, 2007, pp. 39-42.

[5] D. J. Sturman, D. Zeltzer, and S. Pieper, "Hands-on interaction with virtual environments," in ACM SIGGRAPH Symposium on User interface software and technology, New-York, NY, 1989, pp. 19-24.

[6] I. Rosenberg and K. Perlin, "The unmousepad - an interpolating multitouch force-sensing input pad," in ACM SIGGRAPH, 2009, pp. 1-9.

[7] J. Hook, S. Taylor, A. Butler, N. Villar, and S. Izadi, "A reconfigurable ferromagnetic input device," in ACM Symposium on User Interface Software and Technology, 2009.

[8] S. Kim, M. Ishii, Y. Koike, and M. Sato, "Development of tension based haptic interface and possibility of its application to virtual reality," in ACM symposium on Virtual Reality Software and Technology, 2000, pp. 199-205.

[9] M. Bouzit, G. Burdea, G. Popescu, and R. Boian, "The rutgers master ii-new design force-feedback glove," IEEE/ASME Transactions on Mechatronics, vol. 7, p. 2, June 2002.

[10] J. Allard, S. Cotin, F. Faure, P.-J. Bensoussan, F. Poyer, C. Duriez, H. Delingette, and L. Grisoni, "Sofa - an open source framework for medical simulation," in Medecine Meets Virtual Reality, 2007, pp. 13-18.

[11] J.-R. Chardonnet, "Interactive dynamic simulator for multibody systems," International Journal of Humanoid Robotics, to appear.

[12] R. S. Johansson, "Sensory input and control of grip," in Novartis Foundation Symposium, 1998, pp. 45-59.

[13] S. Zhai, P. Milgram, and W. Buxton, "The influence of muscle groups on performance of multiple degree-of-freedom input," in CHI, 1996, pp. 308-315.

[14] B. E. Insko, "Passive haptics significantly enhances virtual environments," University of North Carolina, Tech. Rep., 2001.

[15] A. Lecuyer, S. Coquillart, A. Kheddar, P. Richard, and P. Coiffet, "Pseudo-haptic feedback: can isometric input devices simulate force feedback?" in IEEE Virtual Reality, New Brunswick, NJ, March 18-22 2000, pp. 83-90.

[16] D. K. Pai, E. W. Vanderloo, S. Sadhukhan, and P. G. Kry, "The tango: A tangible tangoreceptive whole-hand human interface." in Joint Eurohaptics Conference and Symposium on Haptic Interfaces for Virtual Environment and Teleoperator Systems, 2005, pp. 141-147.

[17] A. Talati, F. J. Valero-Cuevas, and J. Hirsch, "Visual and tactile guidance of dexterous manipulation tasks: an fmri study," Perceptual and Motor Skills, vol. 101, pp. 317-334, 2005.

[18] E. Wiese, J. H. Israel, A. Meyer, and S. Bongartz, "Investigating the learnability of immersive free-hand sketching," in ACM SIGGRAPH/Eurographics Symposium on Sketch-Based Interfaces and Modeling, Annecy, France, 2010.

[19] S. G. Hart and L. E. Stavenland, "Development of nasa-tlx (task load index): Results of empirical and theoretical research," in Human Mental Workload, P. A. Hancock and N. Meshkati, Eds. Elsevier, 1988, ch. 7, pp. 139-183.

[20] S. M. Kosslyn, G. J. Digirolamo, W. L. Thompson, and N. M. Alpert, "Mental rotation of objects versus hands neural mechanisms revealed by positron emission tomography," Psychophysiology, vol. 35, pp. 151-161, 1998.

[21] I. M. Harris, G. F. Egan, C. Sonkkila, H. J. Tochon-Danguy, G. Paxinos, and J. D. G. Watson, "Selective right parietal lobe activation during mental rotation - a parametric pet study," Brain, vol. 123, pp. 65-73, 2000.

[22] W. Penfield and T. Rasmussen, The Cerebral Cortex of Man: A Clinical Study of Localization of Function. The Macmillan Company, 1950.

[23] R. Balakrishnan and I. S. MacKenzie, "Performance differences in the fingers, wrist, and forearm in computer input control," in CHI, 1997, pp. 303-310. 